\title{
Use of tumour marker immunoreactivity to identify primary site of metastatic cancer
}

\author{
A R Gamble, J A Bell, J E Ronan, D Pearson, I O Ellis
}

\begin{abstract}
Objectives-To determine whether variations in the expression of tumour related antigens can predict the origin of tumours.

Design-Immunohistological study of tumour marker expression in primary adenocarcinomas and respective metastatic deposits. Antibodies to the following tumour markers were used: polymorphic epithelial mucin (NCRC-11 and SM3), carcinoembryonic antigen, carcinoembryonic antigen with non-specific antigen cospecificity, CA125, CA19.9, prostate specific antigens, and thyroglobulin.

Setting-Histopathology department of teaching hospital.

Subjects-100 pathology sections of metastatic adenocarcinoma and their related primary tumours.

Main outcome measures-Concordance of reactivity between primary and metastatic tumours. Reactivity profiles of tumour sites.

Results-The correct primary site of origin was predicted in $70 \%(33 / 47)$ of tumours in men and $54 \%$ (27/43) tumours in women with antibodies SM3, 288, CA19.9, CA125, and PSA (men only). Specificities ranged from $68 \%$ for breast tumour to $98 \%$ for prostate tumour.

Conclusion-Use of tumour markers in patients presenting with metastatic adenocarcinoma of unknown origin can help localise the probable primary sites and reduce the need for extensive and expensive imaging te' hniques.
\end{abstract}

\section{Introduction}

Metastasis of unknown origin is defined as a metastatic tumour, the primary tumour of which remains occult despite taking a clinical history, physical examination, chest radiography, analysis of blood and urine samples, and histological and electron microscopic evaluation. It is a common phenomenon, accounting for $5-10 \%$ of all cancers. ${ }^{1}$ The primary site becomes apparent in $20 \%$ of patients during their lifetime and $75 \%$ are found at necropsy. ${ }^{1}$ About $75 \%$ of occult primary tumours are below the diaphragm, predominantly in the pancreas, colon, stomach, and liver. The lung is the most common site above the diaphragm, accounting for $15-20 \%$ of cases.

Patients presenting with metastasis of unknown origin have a mean life expectancy of only four months so most diagnostic methods occupy a large proportion of the patient's remaining life and identification of the primary site only rarely influences the choice of treatment. Nevertheless, many patients receive extensive and expensive investigations, perhaps more for clinical interest than to benefit the patient. Some patients with metastasis of unknown origin do have responsive tumours. Investigations should therefore aim at identifying patients with tumours that might respond to systemic treatment (either chemotherapy or some form of endocrine manipulation). Any diagnostic system that is quicker and cheaper than the present system would be of use.

Certain tumour markers such as thyroglobulin and prostate specific antigen can provide sensitive and specific diagnostic information about the primary site of metastatic thyroid and prostate tumours. ${ }^{23}$ Many studies have examined the diagnostic potential of immunohistochemistry, ${ }^{4-8}$ but they have generally failed to show the clear definitions found with prostate specific antigen or thyroglobulin. Combinations of such antibodies may offer a more accurate system for identification of tumour type.

We examined the immunoreactivity of a large range of tumour marker antisera in a series of adenocarcinomas and their respective metastases to determine whether patterns of reactivity could help identify the primary sites of metastases and to assess the clinical value of this system as a routine method of investigation. The cases used in this study were selected to include a range of primary sites and were not true cases of metastasis of unknown origin.

\section{Methods}

We searched the computer records of the histopathology department for 1987 to 1991 to identify patients who had had adenocarcinoma or metastatic neoplasm diagnosed. A consecutive series of 1100 patients was identified. We selected 100 patients in this series who had both a known primary site and synchronous or appropriate non-synchronous metastatic disease. Patients were selected consecutively on the basis of primary site of tumour. The numbers of tumours at each site were predetermined according to the approximate frequency of presentation at that primary site. Sites chosen included colon, small intestine, stomach, pancreas, salivary gland, oesophagus, breast, lung, kidney, bladder, endometrium, ovary, fallopian tube, and prostate. The histological sections of each lesion were reviewed to select tissue blocks containing representative and adequate volumes of tumour. In all cases a diagnostic primary tumour block was also reacted with the antibodies.

Twelve sections were cut from each formalin fixed paraffin wax embedded block at a thickness of $3 \mu \mathrm{m}$. The tissue sections were stained by the avidin-biotin complex immunocytochemical method, ${ }^{9}$ with diaminobenzidine as the chromogen and copper sulphate for end product enhancement. Table I shows the primary antisera used.

Each section was examined through a microscope and scored by a single observer (without knowledge of tissue type or site of origin) from zero to four depending on the number of cells reacting. A score of 0 represented no reactivity, 1 represented strong staining in up to $1 \%$ of cells, 2 strong staining in $<30 \%$, 3 staining in $<60 \%$, and 4 staining in up to $100 \%$. For
Correspondence to: Dr Ellis. 
TABLE I-Details of the monoclonal antibodies used in study

\begin{tabular}{|c|c|c|}
\hline Designation & Specificity & Source \\
\hline NCRC-11 & Polymorphic epithelial mucin & $\begin{array}{l}\text { Cancer Research Campaign Laboratories, } \\
\text { Nottingham }\end{array}$ \\
\hline SM3 & Polymorphic epithelial mucin & Imperial Cancer Research Fund, London \\
\hline 228 & Carcinoembryonic antigen & $\begin{array}{l}\text { Cancer Research Campaign Laboratories, } \\
\text { Nottingham }\end{array}$ \\
\hline 198 & $\begin{array}{l}\text { Carcinoembryonic antigen (with non-specific antigen } \\
\text { cospecificity) }\end{array}$ & $\begin{array}{l}\text { Cancer Research Campaign Laboratories, } \\
\text { Nottingham }\end{array}$ \\
\hline CA125 & Ovarian related antigen & CIS UK, High Wycombe \\
\hline CA19.9 & Pancreas related antigen & CIS UK, High Wycombe \\
\hline PSAP & Prostate specific antigen & DAKO \\
\hline PSA & Prostate specific antigen & DAKO \\
\hline Thyroglobin & Thyroid related antigen & DAKO \\
\hline
\end{tabular}

the purposes of the discriminate analysis tissues were classified as showing no reactivity versus any reactivity.

Reactivity to thyroglobulin and the prostatic antigens was not included in the discriminate analysis, as these markers seemed highly sensitive and specific for their respective tumours and therefore would not contribute to the analysis.

We used discriminate analysis for the remaining results, with the primary diagnosis group as the dependent variable and entering the scores for antibody markers stepwise. The discriminant functions obtained were used to predict the site of the primary tumour in the samples and the results compared with the actual primary diagnosis for each patient. Tumours (except thyroid and prostate) were grouped according to their anatomical relation and treatability: breast; lung; gut (including colon, oesophagus, stomach, and small intestine); female genital tract (including ovarian, fallopian tube, and endometrium); and others (including pancreatic, renal, bladder, and salivary gland).

\section{Results}

The intraobserver variability of the scoring method was assessed by repeat scoring of 46 samples. The score was different in nine cases, but the difference was no more than one. The results were then compared by a Wilcoxon signed rank test for match pairs; significance was $0 \cdot 2$, which is satisfactory.

Overall there was no significant difference between the reactivities of primary and secondary tumours for any of the antibodies. Some of the antibodies, NCRC-11 and SM3 (against polymorphic epithelial mucin), 198 and 228 (against carcinoembryonic antigen), and PSAP and PSA (against prostate specific antigen), gave comparable results and in discriminate

TABLE II-Predicted versus actual primary tumour sites in men (10 prostate cases excluded)

\begin{tabular}{lrrrr}
\hline & \multicolumn{5}{c}{ Predicted site $^{\star}$} \\
\cline { 2 - 5 } & Other & Gut & Lung & Total \\
\hline Actual site: & & & & \\
Other & 13 & 1 & 1 & 15 \\
Gut & 5 & 15 & 3 & 23 \\
Lung & 3 & 1 & 5 & 9 \\
\hline Total & 21 & 17 & 9 & 47
\end{tabular}

^Predicted from reactivity of metastatic tumour to CA19.9, 228, SM3, and CA125.

TABLE III-Predicted versus actual primary tumour sites in women

\begin{tabular}{lccccc}
\hline & \multicolumn{5}{c}{ Predicted site $^{\star}$} \\
\cline { 2 - 6 } & Breast & Gut & Gynaecological & Lung & Total \\
\hline Actual site: & & & & & \\
Breast & 6 & 0 & 5 & 1 & 12 \\
Gut & 2 & 8 & 0 & 0 & 10 \\
Gynaecological & 6 & 1 & 9 & 0 & 16 \\
Lung & 2 & 1 & 0 & 2 & 5 \\
\hline Total & 16 & 10 & 14 & 3 & 43
\end{tabular}

$\star$ Predicted from reactivity of metastatic tumour to CA19.9, 228, SM3, and CA125. analysis knowledge of reactivity of both antibodies in each of these pairs did not provide appreciable additional information. The number of antibodies required in the panel was therefore rationalised. The optimum panel for investigation of men comprised PSA, CA19.9, 228, SM3, and CA125 and predicted the primary site for the common types of tumours found in men with an accuracy of $70 \%$ (table II). In women this panel of antibodies (without PSA) gave a level of discrimination between the common groups of tumours of $58 \%$ (table III).

The sensitivity and specificity of the antibody panel for each tumour group were determined from tables II and III. Tumour sites correctly predicted into group $\mathrm{X}$ were classified as true positive results; those incorrectly predicted into group $\mathrm{X}$ as false positive results; those incorrectly not predicted into group $\mathrm{X}$ as false negative results; and those correctly not predicted into group $\mathrm{X}$ as true negative results (table IV).

TABLE IV-Sensitivities and specificities of antibody panel in predicting site of primary tumour

\begin{tabular}{lcc}
\hline Site of primary tumour & Sensitivity (\%) & Specificity (\%) \\
\hline Breast & 50 & 68 \\
Gut & 70 & 93 \\
Gynaecological & 56 & 81 \\
Lung & 50 & 93 \\
Others & 87 & 75 \\
Prostate & 74 & 98 \\
Thyroid & & 100 \\
\hline
\end{tabular}

\section{Discussion}

We have shown that knowledge of immunohistological reactivity with a panel of six antibodies to tumour markers can predict the primary site of origin correctly in $70 \%$ of tumours in men and $58 \%$ of tumours in women with specificities ranging from $68 \%$ for breast tumours to over $90 \%$ for tumours in the gut, lung, prostate, and thyroid. This system should not be judged solely on its ability to diagnose the occult primary site correctly (sensitivity) but also on its potential to exclude other sites. In patients who could potentially benefit from treatment of advanced disease (those with breast, gynaecological, prostate, or thyroid tumours) the specificity was at least $68 \%$ and would be of clinical value. The tests would enable identification (with varying certainty) of patients who might respond to treatment and increase their survival.

The results of the antibody tests were interpreted without knowledge of metastatic site or other clinical information and are considerably better than results of morphological studies using routine sections, ${ }^{10}$ in which only $27 \%$ of sites were identified correctly on morphology alone and $46 \%$ with knowledge of the metastatic site. Electron microscopy may improve identification but it is not widely available, is expensive, and is limited by the need for specially fixed tissues when generally only formalin fixed tissues are available. Although formalin fixed tissue can be used or transferred into glutaraldehyde, the loss in quality of the sample precludes identification of subtle features. Ultrastructural evidence of neuroendocrine differentiation may be shown but is not widely used to discriminate primary site of origin. With the exception of computed tomography most radiological investigations cannot identify the primary tumour in metastatic disease of unknown origin." Gorich et al found the primary site by computed tomography in $58 \%$ of 31 patients with metastatic disease of unknown origin. ${ }^{12}$ Among the remaining patients, computed tomography was helpful in $19 \%$, but unhelpful or wrong in $23 \%$. Serological studies (for the markers $\alpha$ fetoprotein, $\beta$ human chorionic gonadotrophin and carcinoembryonic antigen) are generally of little use 
because of their lack of specificity and are generally used to monitor patients with tumours of known primary site-for example, CA125 in ovarian cancer. ${ }^{\circ}$

Knowledge of the immunohistological profile of a metastatic tumour can be used to determine which patients should receive additional investigations. Interpretation of the metastatic spread of the lesion, symptoms, and history; careful physical examination, computed tomography; mammography; immunoscintigraphy of likely primary sites; and relevant serum assays may also be helpful, but these tests are often expensive. Such investigations could be used more effectively if guidance about the tumour site was available. For example selective computed tomography could be performed instead of whole body scans, which are extremely expensive. Investigative mastectomy could be considered for women with normal mammograms who present with axillary nodal disease when antibody reactivity supports a primary tumour in the breast.

The reproducibility of the immunohistological results was good. A highly sensitive immunohistochemical technique was used (avidin-biotin complex) ${ }^{9}$ and it is unlikely that any improvement on the methodology would be worth while.

\section{CHOICE OF ANTIBODIES}

The value of each antibody as a member of a panel should be considered critically. Many studies have shown that the antibodies PSA and PSAP have over $90 \%$ sensitivity for prostatic adenocarcinomas. ${ }^{3} \mathrm{We}$ found sensitivities of $84 \%$ and $74 \%$ for PSAP and PSA respectively. Another study found that poorly differentiated solid carcinomas showed only weak staining ${ }^{13}$ and in our small sample a poorly differentiated tumour would significantly decrease the apparent sensitivity. Since metastases of unknown origins are usually poorly differentiated the sensitivity of testing with these antibodies would also be reduced. Although we could not determine the sensitivity of thyroglobulin, the specificity of about $100 \%$ agrees with previous results. ${ }^{14}$

The expression of ovarian tumours of the antigen CA125 is dependent on whether the tumour is serous or mucinous. ${ }^{15}$ Serous ovarian tumours are generally consistent and strong expressors of the antigen whereas mucinous tumours may show no or only weak staining. Our antibody panel is therefore useful in identifying serous ovarian tumours but would not identify mucinous ovarian tumours correctly. Further assessment of this problem was not possible because of the small number of cases but knowledge of histological type could further assist in determining the sensitivity of this method. Endometrial reactions with the antibody CA125 have been reported to be $84 \%$ sensitive ${ }^{16}$ in frozen sections. We used paraffin sections which may account for the $50 \%$ sensitivity that we found.

Binding of the antibody SM3 showed a similar pattern to that with NCRC-11. These two antibodies recognise epitopes on the core protein of the same high molecular weight glycoprotein (sometimes called epithelial membrane antigen and recently called polymorphic epithelial mucin), ${ }^{17}$ which is normally expressed by exocrine gland cells and a wide range of adenocarcinomas. ${ }^{18}$ SM3 had a greater specificity for breast and lung tumours than NCRC-11. The coding sequence on the mucin core protein recognised by SM3 is relatively long. It has been suggested that extensive exposure of the core protein occurs more commonly in breast cancer than in other cancers, ${ }^{17}$ which would give SM3 some specificity for breast cancer. NCRC-11 has a smaller coding region and it is therefore more likely to be expressed in other cancers. Our results support this hypothesis.
Reactions with carcinoembryonic antigen antibodies such as 228 are variable and not highly specific, although in our series most gastrointestinal tumours (stomach, colon, small intestine) scored highly with 228. Pancreatic tumours, however, generally showed weak reactivity. These results are similar to those of Heyderman et al, who found that 20 out of 22 primary pancreatic tumours focally or weakly stained for carcinoembryonic antigen.

\section{FUTURE PROSPECTS}

Other antibodies with site specificity will probably be produced or recognised in the future. Such antisera could improve the ability of a panel to identify primary tumour site. It may also be possible to extend the diagnostic discrimination using cytokeratin subtype classification, neuroendocrine status, or serological antibody titres. For example, the new antibodies to progastricsin ${ }^{5}$ and DD9-E7 ${ }^{2}$ could increase specificity of diagnosis of intra-abdominal adenocarcinoma (colon, stomach, small intestine, pancreas). The enzyme progastricsin is present in $30-40 \%$ of gastric adenocarcinomas and is thought to be useful in differentiating between colonic and gastric adenocarcinomas. ${ }^{5}$ Gastric carcinoma is very common in patients presenting with metastasis of unknown origin, although at present the antibody's clinical usefulness is questionable as the disease has a poor prognosis. Antibody to DD9-E7 has been reported to have $100 \%$ sensitivity for adenocarcinomas of the exocrine pancreas. ${ }^{2}$ Further work is needed to establish whether it should be included in an antibody panel.

In conclusion our results show the potential value of immunoreactivity of tumour markers in assisting identification of the site of the primary tumour in patients with metastasis of unknown origin. It is an inexpensive, simple procedure which could be performed as part of the diagnostic histopathological process and could direct or reduce the need for subsequent imaging procedures. If used routinely with data derived from a prospective study, a probability rating for primary site could be determined during confirmation of metastatic adenocarcinoma.

Antibodies 198, 228, and NCRC 11 were gifts from the Cancer Research Campaign Laboratories, Nottingham; SM3 was a gift from Dr J Taylor-Papadimitriou; CA19.9 and CA125 were bought from CIS UK, High Wycombe; PSAP, PSA, thyroglobulin, and the intermediate antibody reagents were bought from DAKO Ltd, High Wycombe.

1 Greco FA, Hainsworth JD. The management of patients with adenocarcinoma and poorly differentiated carcinoma of unknown primary site. Semin Oncol 1989;16(suppl 6):116-22.

2 Heyderman E, Larkin SE, O'Donnell PJ, Haines AM, Warren PJ, Northeast A, et al. Epithelial markers in pancreatic carcinoma: immunoperoxidase localisation of DD9, CEA, EMA and CAM 5.2. I Clin Pathol 1990;43: 448-52.

3 Hammond ME, Sause WT, Martz KL, Pilepich MV, Asbell SO, Rubin P, et al. Correlation of prostate-specific acid phosphatase and prostate-specific et al. Correlation of prostate-specific acid phosphatase and prostate-specific antigen immunocy

4 Keep JC, Benson AB, Sullivan MD, Carney WP, Rosen ST, Radosevich JA. Immunophenotypic analysis of colorectal carcinomas with monoclona antibodies 47D10 and anti-carcinoembryonic antigen. Tumour Bio 1989;10:153-6.

5 Reid WA, Branch T, Gorman C. Identification of primary tumour site by immunolocalization of progastricsin in metastatic adenocarcinoma. $f$ Patho 1990;160:203-7.

6 Rosen A, Sevelda P, Klein M, Spona J, Beck A. A CA125 score as a prognostic index in patients with ovarian cancer. Arch Gynecol Obstet 1990;247:125-9.

7 Sterns EE, Cochran AJ. Monoclonal antibodies in the diagnosis and treatment of carcinoma of the breast. Surg Gynaecol Obstet 1989;169:81-91.

8 Hamaya K, Doi K, Yoshizawa S. An immunohistological study of metastatic adenocarcinoma of the lymph node: is it useful in diagnosing a primary tumour? Gan No Rinsho 1988:34:1956-60.

9 Coggi G, Dell'orto P, Viale G. Avidin biotin methods. In: Polak JM, van Noorden S, eds. Immunohistochemistry: modern methods and applications. 2nd ed. Bristol: Wright, 1986.

10 Sheahan K, Abramowitz A, Carlson JA. Accuracy of pathologic evaluation of metastatic carcinoma of unknown primary site. An analysis using computerized image storage system. Lab Invest 1990;62:112A.

11 Nystrom JS, Weiner J, Wolf R, Bateman JR, Viola MU. Identifying the primary site in metastatic cancer of unknown origin; inadequacy of roentgenographic procedures. $\Im A M A$ 1979;241:381-3.

12 Gorich J, Beyer-Enke SA, Muller M, Guckel F, Probst G, Van Kaick G. The 
value of computed tomography in the search for an unknown primary tumour. ROFO 1988;149:277-9,

$13 \mathrm{Ma} \mathrm{WX}, \mathrm{Li}$ W. Immunohistochemical detection of prostate carcinoma. Chung-hua Ping Li Hsuch 7sa Chih 1989;18:37-9.

14 Sturm U, Grossmann H, Schuh D, Zotler S, Muller M. Immunohistochemical detection of thuroglobulin. Zentralbl Allg Pathol 1989;135:281-5.

15 Hitchcock A, Ellis IO. A combined serological and immunohistological investigation of $\mathrm{CA} 125$ antigen and malignant ovarian epithelial tumours. fObstet Gymaecol 1987:8:173-8.

16 Berchuck A, Soisson AP, Clarke-Pearson DL, Soper JT, Boyer CM, Kinney $\mathrm{BB}$. Immunohistochemical expression of $\mathrm{CA} 125$ in endometrial adeno- carcinoma: correlation of antigen expression with metastatic potential. Cancer Res 1989;49:2091-5.

17 Gendler S, Taylor-Papadimitriou J, Dubig T. A highly immunogenic region of a human polymorphic epithelial mucin expressed by carcinomas made up of tandem repeats. F Biol Chem 1988;263:12820-3.

18 Ellis IO, Robins RA, Elston CW, Blamey RW, Ferry B, Baldwin RW. A monoclonal antibody, NCRC 11, raised to human breast carcinom. 1. Production and immunohistological characterisation. Histopatholo 1984;8:501-16

(Accepted 26 November 1992)

\title{
Who's afraid of informed consent?
}

\section{D Kerrigan, R S Thevasagayam, T O Woods, I Mc Welch, W E G Thomas, A J Shorthouse, A R Dennison}

\begin{abstract}
Objective-To test the assumption that patients will become unduly anxious if they are given detailed information about the risks of surgery in an attempt to obtain fully informed consent.

Design-Preoperative anxiety assessed before and after patients were randomly allocated an information sheet containing either simple or detailed descriptions of possible postoperative complications.
\end{abstract}

Setting-Four surgical wards at two Sheffield hospitals.

Subjects-96 men undergoing elective inguinal hernia repair under general anaesthesia.

Main outcome measure-Change in anxiety level observed after receiving information about potential complications.

Results-Detailed information did not increase patient anxiety (mean Spielberger score at baseline $33.7(95 \%$ confidence interval 31.3 to $36 \cdot 2)$, after information $34.8(32.1$ to $37 \cdot 5) ; p=0 \cdot 20$, paired $t$ test). A simple explanation of the facts provided a statistically significant degree of reassurance (mean score at baseline $34.6(31.5$ to 37.6$)$, after information $32.3(29.8$ to 34.9$) ; p=0.012)$, although this small effect is likely to be clinically important only in those whose baseline anxiety was high $(r=0.27, p=0.05)$.

Conclusions-In men undergoing elective inguinal hernia repair a very detailed account of what might go wrong does not increase patient anxiety significantly and has the advantage of allowing patients a fully informed choice before they consent to surgery, thus reducing the potential for subsequent litigation.

Royal Hallamshire Hospital, Sheffield S10 2JF D D Kerrigan, lecturer R S Thevasagayam, medical student

I Mc Welch, house officer W E G Thomas, consultant surgeon

A J Shorthouse, consultant surgeon

A R Dennison, senior registrar

Department of Child and Adolescent Psychiatry, Booth Hall Children's Hospital, Manchester M9 2AA

T O Woods, senior registrar

Correspondence to: Mr D D Kerrigan, Department of Surgery, Royal Oldham Hospital, Oldham OL1 2JH

BMF 1993;306:298-300

\section{Introduction}

The NHS Management Executive's recent guidance on obtaining consent from patients is a pertinent reminder of the importance with which the government views our legal requirement to obtain fully informed consent from patients undergoing treatment. ${ }^{1}$ In the unhappy event of litigation, a signed consent form may be disregarded by the courts unless it can be shown that the patient was "given sufficient information, in a way they can understand, about the proposed treatment." Sadly, the standards of consent actually achieved on the ward often fall short of those expected by lawyers, perhaps because the task of obtaining consent is left to more junior medical staff, who are themselves ignorant of many of the potential pitfalls that might face the patient. It has been estimated that every year about 300000 patients in the United Kingdom experience some form of harm as a result of being admitted to hospital, ${ }^{2}$ and if claims for medical negligence are to be minimised it is vitally important that doctors ensure that the patient has carefully considered the potential risks of any procedure as well as its likely benefits.

One solution is to adopt the North American practice of providing patients with a comprehensive list of postoperative complications, but to most British doctors the prospect of burdening patients with "unwanted" information about what might go wrong is deemed to be both unhelpful and unkind. In view of this dichotomy of opinion it is surprising that there is little, if any, objective evidence to support or refute the rather paternalistic British view that allowing patients to make a fully informed decision about their treatmen would generate an unnecessary and harmful degree of anxiety. The aim of this study was to find out who is really afraid of fully informed consent: British patients or their doctors?

\section{Subjects and methods}

Ninety six male patients admitted to four surgical wards for elective repair of inguinal hernias under general anaesthesia were interviewed in hospital on the day before surgery and asked to complete two self evaluation questionnaires: a screen for pre-existing anxiety or depressive states using the hospital anxiety and depression scale (HADS), ${ }^{3}$ in which patients were asked to score answers based on how they generally fel over the few weeks before admission, and an assessment of their current state of anxiety (Spielberger STAI-X1). The Spielberger anxiety scale consists of twenty statements that gauge how respondents feel "right now, at this moment" and has been widely evaluated in healthy American adults and in nonpsychiatric hospital inpatients. ${ }^{+}$Subjects chose from one of four graded responses to each statement, generating a total score between 20 (low anxiety) and 80 (very high anxiety). After this baseline assessment was obtained patients were randomly allocated one of two typed information sheets which contained a simple description of what a hernia is, why surgery was necessary, and what the operation entailed. The difference between the two information sheets was that one provided a rather sketchy outline of possible postoperative complications (derived from a survey of what 10 house officers actually told hernia patients when they obtained consent), and the other contained a more comprehensive list (boxes). To prevent patients on the same ward comparing the contents of different fact sheets (and thereby contaminating the data), randomisation depended on the ward and week of admission: forms used on a given ward were randomly alternated each week.

To standardise the manner in which information was presented by the investigator, patients were simply left to digest the written information for one hour, but they were given the opportunity to ask the investigator to 